\title{
MECHANISM OF CYCLOTRON RESONANCE INDUCED CONDUCTIVITY IN $n$-GaAs
}

\author{
H.J.A. Bluyssen, J.C. Maan, L.J. van Ruyven*, F. Williams† and P. Wyder \\ Research Institute for Materials, University of Nijmegen, Toernooiveld, Nijmegen, The Netherlands
}

(Received 6 October 1977 by A.R. Miedema)

\begin{abstract}
A detailed mechanism is proposed and varified experimentally for Cyclotron Resonance Induced Conductivity (CRIC, cross modulation) at high magnetic inductions and low electric fields. The principal feature is thermal re-equilibration from donors to optically-depleted Landau levels, thus increasing carrier density. Cyclotron Resonance Induced Hall-effect (CRIH) measurements on $n$-type GaAs prove that CRIC originates from changes in carrier density and not in mobility as previously proposed. This mechanism also explains the observed temperature dependence of CRIC.
\end{abstract}

IT IS WELL KNOWN that cyclotron resonance absorption of radiation by the free carriers of a semiconductor may have a pronounced effect on the conductivity especially for low temperatures. This effect is known as "cross-modulation" or Cyclotron Resonance Induced Conductivity (CRIC) and was used and described by several authors [1-4] as a very sensitive method to detect cyclotron resonance in semiconductors. Sometimes a decrease, sometimes an increase of resistivity is found. Although a number of suggestions about the underlying mechanism have been given, the effect has never been analyzed and understood in detail. It is the purpose of this paper to present a detailed mechanism of CRIC for a simple system, which exhibits an increase in conductivity only, and to verify it experimentally. As a mechanism we assume a thermal equilibration from donors to optically-depleted Landau levels, thus increasing the carrier density. We verify this mechanism in GaAs by means of Cyclotron Resonance Induced Hall Effect (CRIH)-, photoconductivity (CRIC)-, and power transmission (PT)measurements. As a check, CRIC and PT are studied as a function of temperature as well. Though the effect has been observed in several different semiconductors like $\mathrm{Ge}[3,4], \mathrm{AgBr}[5], \mathrm{CdTe}[6]$, and $\mathrm{GaAs}$ [7], we have chosen for our measurements $n$-type GaAs, because of its simple conduction band structure, i.e. a single direct minimum and an isotropic effective electron mass $\left(m^{*}=0.068 m_{0}\right)$. The PT- and CRICmeasurements were performed on the same sample (free carrier density: $n=1.63 \times 10^{15} \mathrm{~cm}^{-3}$; mobility:

* Philips' Research Laboratory, Eindhoven, The Netherlands.

$\dagger$ Department of Physics, University of Delaware, Newark, DE 19711, U.S.A. $\mu=7610 \mathrm{~cm}^{2} / \mathrm{Vsec}$ at $T=300 \mathrm{~K}$; thickness $19 \mu \mathrm{m}$ ), while the CRIH was measured on an especially made Hall-sample $\left(n=1.50 \times 10^{15} \mathrm{~cm}^{-3} ; \mu=7204 \mathrm{~cm}^{2} / \mathrm{Vsec}\right.$ at $T=300 \mathrm{~K}$; thickness $14.3 \mu \mathrm{m}$ ) of the same material. Extrinsic thermal carriers and low electric fields were used, thus avoiding complications arising from photocreated carriers and from excitations due to high electric fields.

To characterize the material, standard Hall measurements were carried out as a function of temperature and the magnetic field strength. All our CR-measurements were done in the temperature range from $10-30 \mathrm{~K}$ and at magnetic inductions of $10.5 \mathrm{~T}$, corresponding to a resonant radiation of $\lambda=70.6 \mu \mathrm{m}$ and an effective mass of $0,069 \mathrm{~m}_{0}$. This far infrared radiation was obtained from an optically pumped $\left(\mathrm{CO}_{2}\right) \mathrm{CH}_{3} \mathrm{OH}$ laser of which the output of $2 \mathrm{~mW}$ in the $70.6 \mu \mathrm{m}$ line was attenuated to about $20 \mu \mathrm{W}$ at the sample. These values for the field $B$ and the temperature $T$ ensure that the necessary conditions for "high field Hall effect" (Hall factor $r_{H}=1$ [8]) and negligible occupation of the $N \neq 0$ Landau levels $\left(\hbar \omega_{c} \gg k T\right)$ are fulfilled.

In general, a change $\Delta \sigma$ in the conductivity $\sigma$ due to the absorbed power could arise from differences in mobilities among the different Landau levels, or from a change $\Delta n$ in the total number of charge carriers in all the Landau levels. Because of $\hbar \omega_{c} \gg k T$, the carriers present in the $N=1$ Landau level due to thermal excitation can be neglected. Defining $n_{0}$ as the thermal equilibrium density of carriers in the $N=0$ Landau level, $n_{1}$ as the density of the carriers in the $N=1$ level due to steady-state cyclotron resonance absorption, $\Delta \mu$ as the difference in mobilities of the $N=1$ and $N=0$ levels, and $\mu_{0}$ as the mobility for the $N=0$ level, we have $\frac{\Delta \sigma}{\sigma}=\frac{\Delta n}{n_{0}}+\frac{n_{1} \Delta \mu}{n_{0} \mu_{0}}$. 

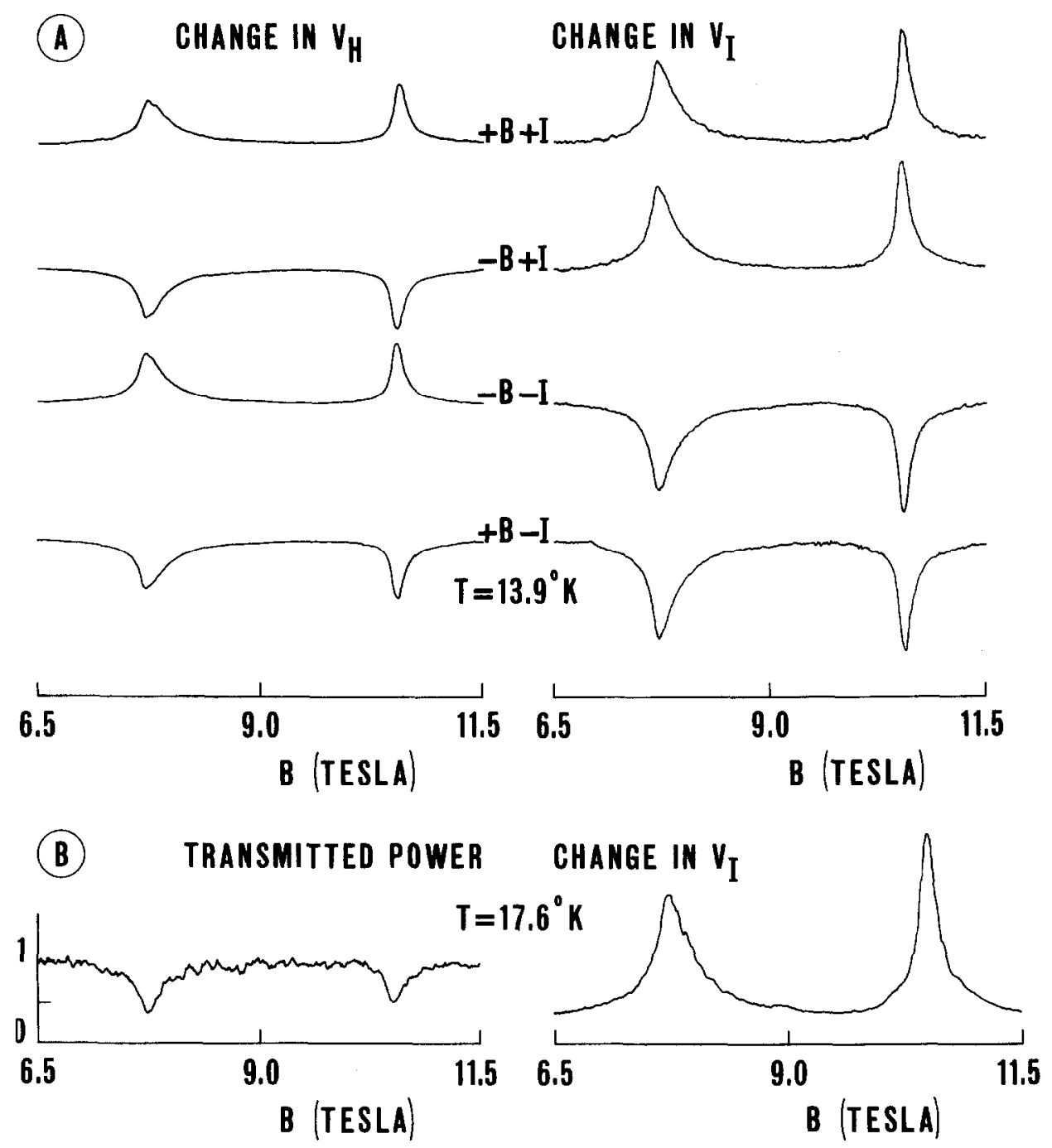

Fig. 1.(a) Typical recorder tracings of Cyclotron Resonance Induced Hall effect $\left(V_{H}\right)$ and Cyclotron Resonance Induced Conductivity $\left(V_{I}\right)$ for different polarities of the magnetic induction $B$ and the current $I$. (b) Power absorption and change in conductivity. The peak at lower fields is the $1 s-2 p^{+}$donor transition, the peak at higher fields is due to cyclotron resonance.

The first term describes the effect from a change in carrier density; the second from a mobility difference. To separate these two possible effects, CRIH was measured since in the high magnetic field limit $\left(r_{H}=1\right)$ the Hall voltage is determined by the number of carriers only.

Typical far infrared PT-, CRIH-, and CRIC-measurements as a function of the magnetic field $B$ are given in Fig. 1 , showing the free carrier cyclotron resonance transition together with the $1 s-2 p^{+}$Zeeman transition at respectively 10.52 and $7.74 \mathrm{~T}$. This last value is in good agreement with a linearly extrapolated value from previous observations at longer wavelengths [9]. The CR transition corresponds to an effective mass of $(0.069 \pm 0.001) m_{0}$, in good agreement with the generally accepted value of $0.068 m_{0}$ [7]. The Hall effect measurements [Fig. 1(a)] show that a true cyclotron resonance induced Hall effect is observed. Since the Hall measurements have been carried out in the high magnetic induction limit, this effect can only arise from an increase in the free carrier density. Finally, since the ratio of the peak amplitudes of the $1 s-2 p^{+}$and CR signal is within our measuring accuracy, the same for $\mathrm{CRIH}$ and CRIC, and since the $1 s-2 p^{+}$transition is well known to be due to a simple carrier excitation, it must be concluded that CRIC involves a change in the number of carriers only. At this point it may be noted that the absence of the mobility differences is somewhat surprising, considering that a number of authors $[2,4]$ have proposed energy-dependent mobility as the dominant mechanism. However, a careful consideration of Halland drift-mobilities (which are equal at these high magnetic fields) compared with the velocity of the electron in the cyclotron orbit reveals that only the 
latter is strongly dependent on Landau level. This distinction may reconcile the discrepancy between mobilities derived from resonance line width and those from Hall measurements [7] as well.

The change of the number of free carriers due to cyclotron resonant absorption can be calculated in the following way. In the absence of the far infrared radiation thermal equilibrium exists between electrons in donor states and electrons in the $N=0$ Landau level (as noted earlier, thermal occupancy of $N=1$ is neglected). The far infrared absorption creates carriers in $N=1$, at the expense of carriers in $N=0$; however, thermal re-equilibration will tend to replenish the population of carriers in the $N=0$ level. A steady-state distribution among Landau- and donor-levels develops, which can be analyzed by detailed balance. The following solution is obtained from combining results with and without the cyclotron resonance absorption:

$\Delta n=n_{1} \frac{\left(N_{D}-N_{A}\right)-n_{0}}{N_{D}-N_{A}}$

where $N_{D}$ and $N_{A}$ are donor and acceptor concentrations. $n_{1}$ is the number of excited carriers, proportional to the absorbed power $\boldsymbol{P}_{\text {abs }}$ and the $\tau$, the lifetime of the $N=1$ Landau level (assuming that the radiation density is low enough so that only spontaneous emission occurs) The peak power absorption for cyclotron resonance transitions is given by

$P_{\text {abs }}=P_{0}\left[1-\mathrm{e}^{-C_{n} / \Delta B}\right]$

where $P_{0}$ is the incident power, $n$ is the total free electron density, $\Delta B$ is the width at half maximum in units of magnetic induction, and $C$ is a proportionality constant, which includes among other things the transition matrix element and the sample thickness.

The oscillator strength is assumed to be independent of temperature. We combine equation (2), after substituting $n_{1}$, and equation (3) to give

$\Delta \sigma=A\left[1-\mathrm{e}^{-C n_{0} / \Delta B}\right] \frac{\left(N_{D}-N_{A}\right)-n_{0}}{N_{D}-N_{A}}$

where $A$ collects constants. The constant $C$ was determined from the temperature dependence of power transmission, and the measured number of carriers from the Hall measurements; only $A$ is left as an adjustable parameter which was fitted to the experimental data of $\Delta \sigma$, The result is shown in Fig. 2. The mechanism explains the essential characteristics of the temperature dependence of $\Delta \sigma$, i.e. an increase below $20 \mathrm{~K}$ due to the increased power absorption, and a decrease above $20 \mathrm{~K}$ due to the fact that not every excited carrier is replenished because of exhaustion of the donors. The derivation of equation (4) is based on neglecting

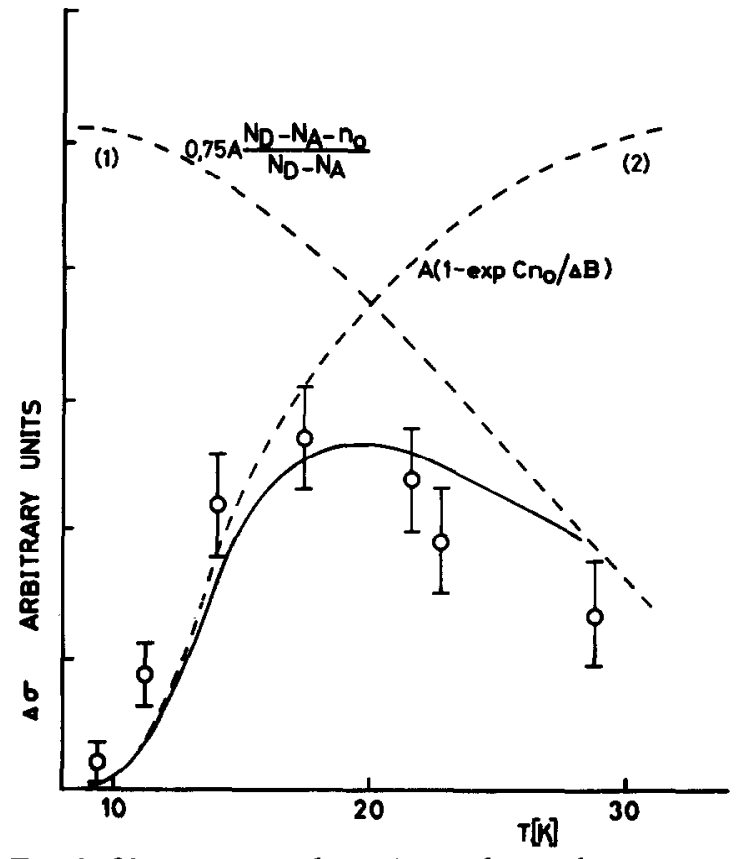

Fig. 2. Change in conductivity under cyclotron resonance conditions in $n-G a A s$ as a function of temperature. The theoretical model (solid line) was fitted to the data using $A$ as a parameter. The result is a product of two thermal effects.shown separately as the dotted lines.

(1) Donor exhaustion, neglecting temperature dependence of the absorption coefficient, taking ( 1 - exp $C n_{0} / \Delta B$ ) for illustrative purposes arbitrarily as 0.75 .

(2) Increasing carrier replenishment due to the increasing absorption coefficient, taking $\left(N_{D}-N_{A}-n_{0}\right) /$ $\left(N_{D}-N_{A}\right)$ as unity.

absorption among the higher Landau levels $N \geqslant 1$ (which is expected to reduce $\Delta \sigma$ at the higher temperatures), and from assuming that $\mu$ and $\tau$ (as well as the radiative transition matrix element below $14 \mathrm{~K}$ ) are temperatureindependent.

We also note that the proposed mechanism explains the high sensitivity of cyclotron resonance induced conductivity at low temperatures. The final factor of equation (4), obtained from equation (3), may be then taken as unity and $\Delta \sigma / \sigma$ is maximal and constant, independent of $n_{0}$. The physical reason for this is that for low absorption both absorption and conductivity depend linearly on $n_{0}$. Because of the lack of phonons with high enough energies, the replenishment time becomes long at low temperatures leading to a small $n_{0}$. Therefore the same temperature dependence is found in the denominator as well as in the numerator causing $\Delta \sigma / \sigma$ to be independent of temperature, or stated differently of the number of carriers.

In conclusion, for $n$-GaAs in high magnetic fields cyclotron reasonance induced conductivity (cross modulation) for $n$-GaAs originates from the change in 
carrier density: thermal replenishment of the opticallydepleted lower Landau levels explains the increase in carrier density and its temperature-dependence, and the transport mobility is found to be independent of the Landau level. Measurements of CRIH and CRIC on the same sample will allow a determination of the individual transport mobilities of the lowest Landau levels. As indicated by our model, it seems to be possible to measure directly the times for thermal equilibration with pulsed CRIC experiments.

We are pleased to thank A. van Etteger and B. Tan for their valuable contributions to the experimental part of this work and C.P. Damen of the Philips' Research Laboratories for preparing the samples. Part of this work has been supported by the "Stichting voor Fundamenteel Onderzoek der Materie" (FOM).

\section{REFERENCES}

1. ZEIGER H.J., RAUCH C.J. \& BEHRNDT M.E., J. Phys. Chem. Solids 8, 496 (1959).

2. LAX B. \& MAVROIDES J.G., Solid State Physics (Edited by SEITZ F. \& TURNBULL D.), Vol. 11, 261 (1960).

3. KAPLAN R., Solid State Commun. 3, 35 (1965).

4. GESHENZON E.M., GURVICH Yu.A., ORLOVA S.L. \& PTITSYNA M.G., Sov. Phys.-Solid State 10, 144 (1968).

5. HODBY J.W., CROWDER J.G. \& BRADLEY C.C., J. Phys. C: Solid State Phys. 7, 3033 (1974).

6. MEARS A.L. \& STRADLING R.A., Solid State Commun. 7, 1267 (1969).

7. CHAMBERLAIN J.M. \& STRADLING R.A., Solid State Commun. 7, 1275 (1969).

8. STILLMAN G.E., WOLFE C.M. \& DIMMOCK J.O., J. Phys. Chem. Solids 31, 1199 (1970).

9. STILlMAN G.E., WOLFE C.M. \& DIMMOCK J.O., Solid State Commun. 7, 921 (1969). 